\title{
X-Ray Reflectometry Study of Self-Assembled Ionic Nanolayers
}

\author{
Szymon Jasiecki, ${ }^{1}$ Jarosław Serafińczuk, ${ }^{2}$ Teodor Gotszalk, ${ }^{2}$ and Grzegorz Schroeder ${ }^{1}$ \\ ${ }^{1}$ Faculty of Chemistry, Adam Mickiewicz University, Grunwaldzka 6, 60-780 Poznań, Poland \\ ${ }^{2}$ Faculty of Microsystem Electronics and Photonics, Wrocław University of Technology, Z. Janiszewskiego 11/17, \\ 50-372 Wrockaw, Poland \\ Correspondence should be addressed to Szymon Jasiecki, szymonj@amu.edu.pl
}

Received 12 October 2011; Accepted 17 January 2012

Academic Editor: Bohua Sun

Copyright () 2012 Szymon Jasiecki et al. This is an open access article distributed under the Creative Commons Attribution License, which permits unrestricted use, distribution, and reproduction in any medium, provided the original work is properly cited.

The self-assembly technique has been applied for the fabrication of thin films including macrocyclic molecules. These multilayered structures, grown by sequential deposition of oppositely charged molecules, were characterised with X-ray reflectometry. The data obtained indicate regular thickness of ion pair layers formed regardless of the number of depositions made as well as the number of ion groups occurring in the molecule. Savitzky-Golay algorithm was used for the calculation of the layer thickness. Formation of self-assembled multilayers (SAMs) occurs not only for polymeric structures but also for small ionic compound systems and results from the electrostatic interaction of many strongly dissipated charges on the whole structure of the molecule.

\section{Introduction}

Self-assembled multilayers (SAMs) have become of great interest since they were reported by Decher and Hong in the early 1990s [1, 2]. The self-assembly process based on electrostatic attraction can be used to obtain multilayered thin film materials showing unique properties [36]. The electrostatic self-assembly (ESA) technique is based on sequential deposition of oppositely charged molecules (polymers) on the substrate surface to form a thin film. Electrostatic self-assembly occurs not only on the solid surface but also in the solution [7] as well as in the air/water interface [8]. ESA is a very simple and versatile method of fabrication of nanoscale thin films. In the past two decades much effort has been made to achieve and improve desired properties of these materials. The role of electrostatic and secondary interactions including hydrophobicity, hydrogen bonding, dispersion forces, ion dipole, and van der Waals interactions has been investigated. Extrinsic conditions are also fundamental, and varying $\mathrm{pH}$ or ionic strength of aqueous solution alters the thickness of layers and the nature of thin film obtained $[5,6]$.

Layer-by-layer (LBL) film structures are promising for commercial applications. Incorporation of metal or inorganic particles leads to interesting electrooptical or magnetic properties [9] of the material potentially applicable in magnetic recording or as memory. The use of LBL films for modification of surface wettability, adhesion, surface conductivity [10], ion transport, and catalytic [11, 12] or specific binding activity [13] illustrates a wide range of their applications in industry. Permeability is an especially interesting property of the LBL films. It is crucial for applications such as drug encapsulation with controlled release or membrane separation [14]. Encapsulation of a drug molecule is connected with the approach to ESA technique that, instead of a planar substrate, colloidal particles have been coated [15]. Subsequent core removal leads to hollow capsules which are spherical closed thin multilayers [3-5].

It has been reported that ionic liquids consisting of multivalent small molecules can create an extended network through pairwise interactions. They form supramolecular polymers, which is in good agreement with the viscosity of such ionic liquids higher than that of cationic or anionic molecules combined with monovalent counter ions [16]. Macrocyclic compounds employed in this experiment are expected to form supramolecular structures with oppositely multicharged polymers or macrocycles. Formation of thin films formed by sequential deposition of oppositely charged polyelectrolyte and small molecules is reported [17-20]. 


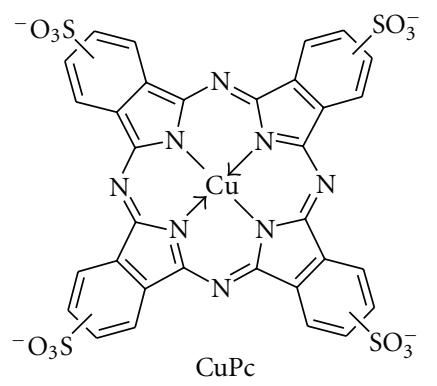

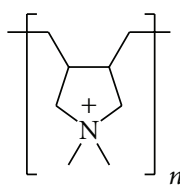

PDDA

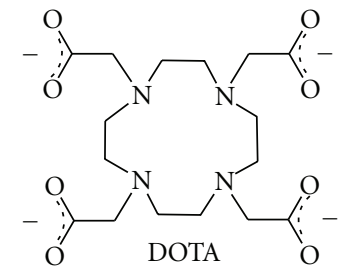<smiles>CC(=O)c1c(C(=O)O)c(C(=O)O)c(C(=O)O)c(C(=O)O)c1C(=O)O</smiles>

MA

FIGURE 1: The structures of the compounds studied.

In this work formation of nonpolymeric ionic nanolayers consisting of small molecules with four or six charged functional groups has been studied by X-ray reflectometry measurements $[15,21]$ utilizing Savitzky-Golay algorithm for the obtained XRR curves [22].

\section{Experimental}

2.1. Functionalization of Surface. The chemical structures of the compounds studied are shown in Figure 1. Positively charged polymer, PDDA, poly(diallyldimethylammonium hydrochloride), and HACO (1,4,7,10,13,16-hexaazacyclooctadecane hexahydrochloride) were deposited as the first layer because of the negative charge of the native oxide on the silicon surface. The negatively charged layer-building blocks were such macrocycles as copper(II) phthalocyanine tetrasulfonic acid tetrasodium salt (CuPc), 1,4,7,10tetraazacyclododecane-1,4,7,10-tetraacetic acid tetrasodium salt (DOTA), and mellitic acid hexasodium salt (MA). Azacyclic compounds as cyclen [23] and hexacyclen are known ligands for cations [24] as well as anions if protonated [25]. Metal phthalocyanines are known for their photochemical [26] and conductivity [27] properties.

Silicon plates with oxidized surfaces were cleaned with methanol in an ultrasonic cleaner and subsequently rinsed with deionized water. Then the plates were dipped for 5 minutes in the PDDA (or HACO resp.) aqueous solution of a concentration of $10^{-3} \mathrm{moldm}^{-3}$. As the positively charged polymers display an attraction to the negatively charged silicon surface with hydroxyl groups, a monolayer of PDDA (or HACO) was expected to be formed on the surface of the plate. Afterwards the plates were washed with deionized water to remove all nonadsorbed polymers. After washing, the plates were immersed in MA (or CuPc or DOTA resp.) aqueous solution of $10^{-3} \mathrm{moldm}^{-3}$ for the next 5 minutes and subsequently washed with deionized water. Consistently a monolayer of negatively charged MA (or CuPc or DOTA) ions was expected to be dispersed and adsorbed on the PDDA (or HACO)-coated sample. After one dipping cycle including one immersion in PDDA (or HACO) solution followed by one immersion in MA (or CuPc or DOTA solution), a bilayer consisting of PDDA (or HACO) and MA (or CuPc or DOTA) monolayers was expected to be created on the sample's surface. Such a procedure was repeated for 5,10 , $15,20,25,30,40$, and 50 times to form a thin film since the opposite charges of PDDA (or HACO) and MA (or CuPc or DOTA) attract these components and ensure their bonding.

The thin films formed on silica surfaces were examined by the X-ray reflectometry.

2.2. X-Ray Reflectometry. The ion nanolayers were investigated by means of high-resolution X-ray diffractometer supported with $\mathrm{Ge}(220)$ four-crystal Bartelss monochromator in the incidence beam, open detector with $0.45 \mathrm{~mm}$ slit in the diffracted beam, and $\lambda_{\text {CuKff1 } 1}=1.540597 \AA$ radiation. Xray reflectivity is a precise and nondestructive method for the determination of thickness, density, and roughness of a layer on a substrate. The method enables observation of layer thickness of the order of angstroms. In X-ray reflectometry measurements, the X-ray beam is directed onto the sample surface. The angle of incidence beam is above the critical angle for investigated material. Below this angle the X-ray beam is totally reflected. In proper measurement the Xrays are reflected from the air/layer as well as from the layer/substrate interfaces. As a result the reflectivity curves with characteristic oscillations are obtained.

The position and number of the oscillations are used to plot a linear function whose directional parameter is used for the calculation of the layer thickness. In this work Savitzky-Golay algorithm was used to reduce noise of the measured curve. The application of this method, widely used in spectroscopy, considerably improves data analysis [22]. 
TABLE 1: The thicknesses of the measured ionic nanolayers.

\begin{tabular}{lcccc}
\hline \multirow{2}{*}{ Quantity of ionic layers } & & \multicolumn{2}{c}{ The thickness of ionic layers [A] } \\
& Series A PDDA and CuPc & Series B PDDA and DOTA & Series C PDDA and MA & Series D HACO and MA \\
\hline 5 & 17.0 & 16.7 & 17.5 & 17.5 \\
10 & 17.0 & 16.7 & 17.1 & 17.5 \\
15 & 17.1 & 16.6 & 18.5 & 17.2 \\
20 & 16.9 & 15.8 & 16.8 & 17.1 \\
25 & 17.0 & 16.6 & 17.5 & 17.2 \\
30 & 17.0 & 16.8 & 17.5 & 17.9 \\
40 & 16.8 & 17.4 & 17.5 & 17.3 \\
50 & 17.2 & 16.4 & 17.5 & 17.2 \\
\hline The value average & 17.0 & 16.6 & \\
\hline
\end{tabular}

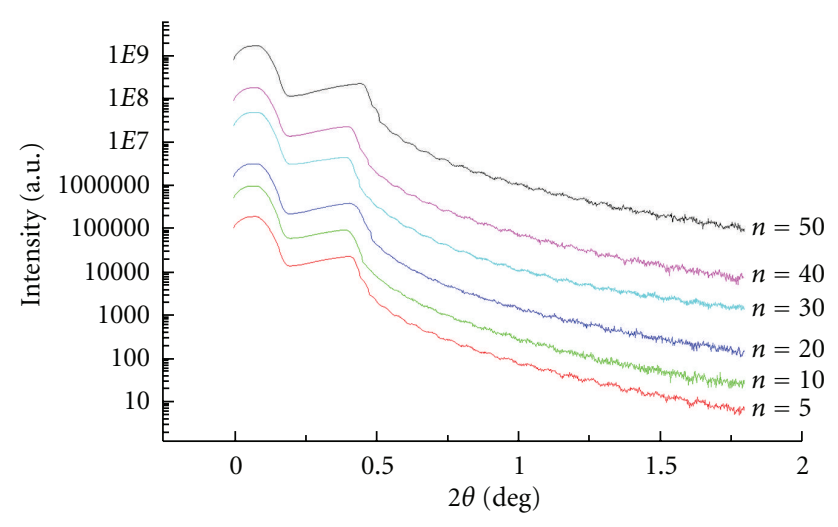

FIgURE 2: Reflectivity curves from the measured ionic nanolayers.

\section{Results and Discussion}

In this work two systems were applied: in the first, the layer deposited on the silicon substrate as well as further positively charged layers were made of PDDA polymer, while in the second the protonated cyclic amine, HACO, was used. A comparison of results obtained for these two systems allowed evaluation of the possibility of SAMs formation when the alternating layers are isolated multicharged molecules instead of polymers.

As prepared thin films were examined by the X-ray reflectometry. The exemplary reflectivity curves obtained for PDDA and $\mathrm{CuPc}$ system are shown in Figure 2. The thicknesses of bilayers for all studied ionic SAM are collected in Table 1. The average values of the layer thicknesses, varying in each series not more than about $10 \%$, indicate a regular thin film growth. Lütt et al. [17] studied the formation of self-assembled thin films of cationic polymer PDDA and NiPc by X-ray refractivity method. They have proposed a hypothetical structure of a thin-film made by PDDA and NiPc. The thickness of a PDDA-NiPc bilayer is of about $8 \AA$.

The results exhibit larger thickness of bilayers deposited at the beginning than those deposited after many depositions. The average layer thickness decreased from about $20 \AA$ for first two immersion cycles to an average and constant value of about $8 \AA$ after deposition of about 40 bilayers. The authors explain a constant increase in the film thickness as well as a constant increase in the roughness of the growth surface by incomplete and random covering of the substrate surface by blocks representing a bilayer.

In our work we studied an analogous system containing $\mathrm{Cu}^{2+}$ ions in the phthalocyanine ring instead of $\mathrm{Ni}^{2+}$ ions. For this system the average thickness of a bilayer was determined as $17.0 \AA$. Such a value indicates that ionic molecules form self-assembled flat layers, in which dissipated charges determine the thickness of a bilayer by electrostatic interaction (Figure 3(a)). The molecules deposited should be lying down as presented in Figures 3(a)-3(d): otherwise, the thickness values would be higher.

The application of multicarboxyl acids salts (MA and DOTA) and ionic polymer PDDA in fabrication of ionic layers confirmed an analogous character of the emerging SAM bilayers as that of the CuPc or NiPc layers [17]. The thickness of these layers depends on the anion used. For an MA system the average thickness of a bilayer is $17.5 \AA$, whereas for a DOTA system this value decreased to $16.6 \AA$. The differences are determined by the lability of carboxyl groups induced by the presence of alkyl linkers. These bilayers are closer packed than the other systems studied. Figures 3(b)-3(c) present visualization of ionic thin film structure as obtained from $\mathrm{x}$-ray measurements.

In this work formation of ionic layers by nonpolymeric ions HACO and MA was also studied. The SAM character of the HACO and MA system is analogous to that of the other systems studied. Reflectivity curves enabled determination of the layers thicknesses for this type of SAM. The average thickness of a bilayer was $17.2 \AA$. Figure $3(\mathrm{~d})$ presents visualization of ionic thin-film structure as obtained from Xray measurements.

\section{Conclusions}

Ionic SAMs on silicon substrate require no polymer component. Formation of layers results from the electrostatic interaction of many strongly dissipated charges on the whole structure of the molecule. Strong localization of charge solely on one functional group prevents from formation of the 


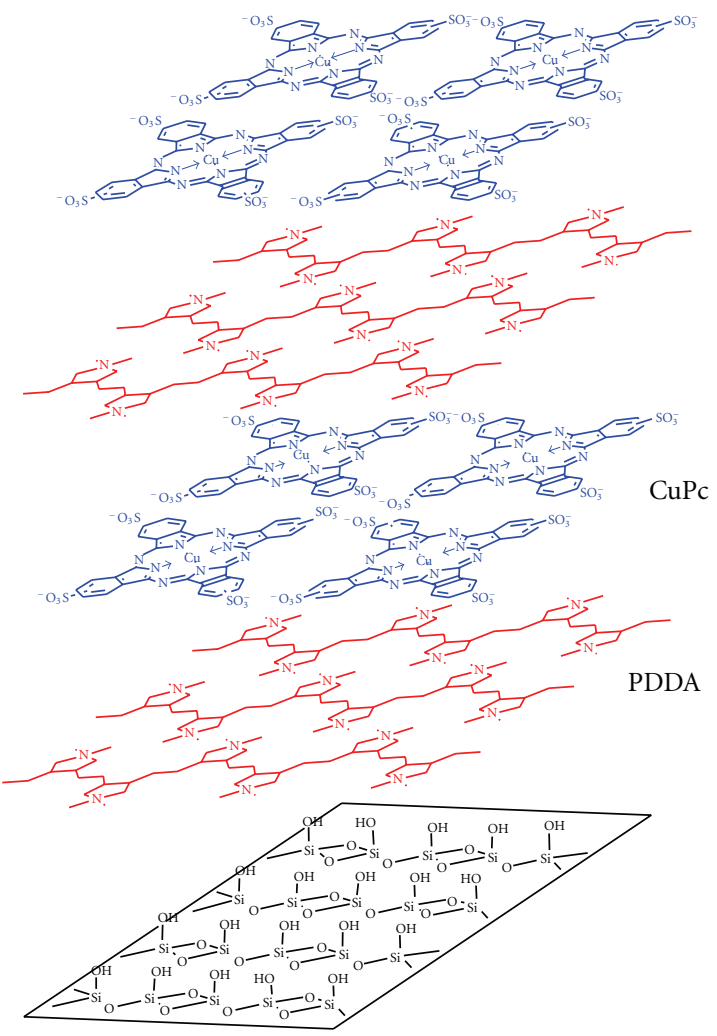

(a)

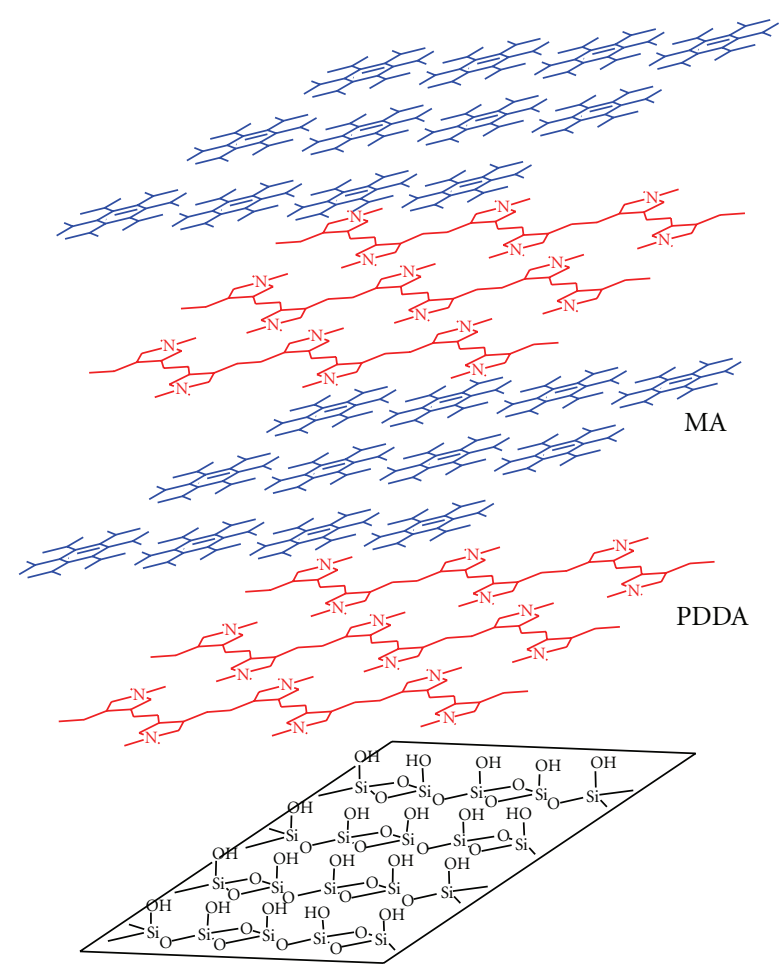

(c)

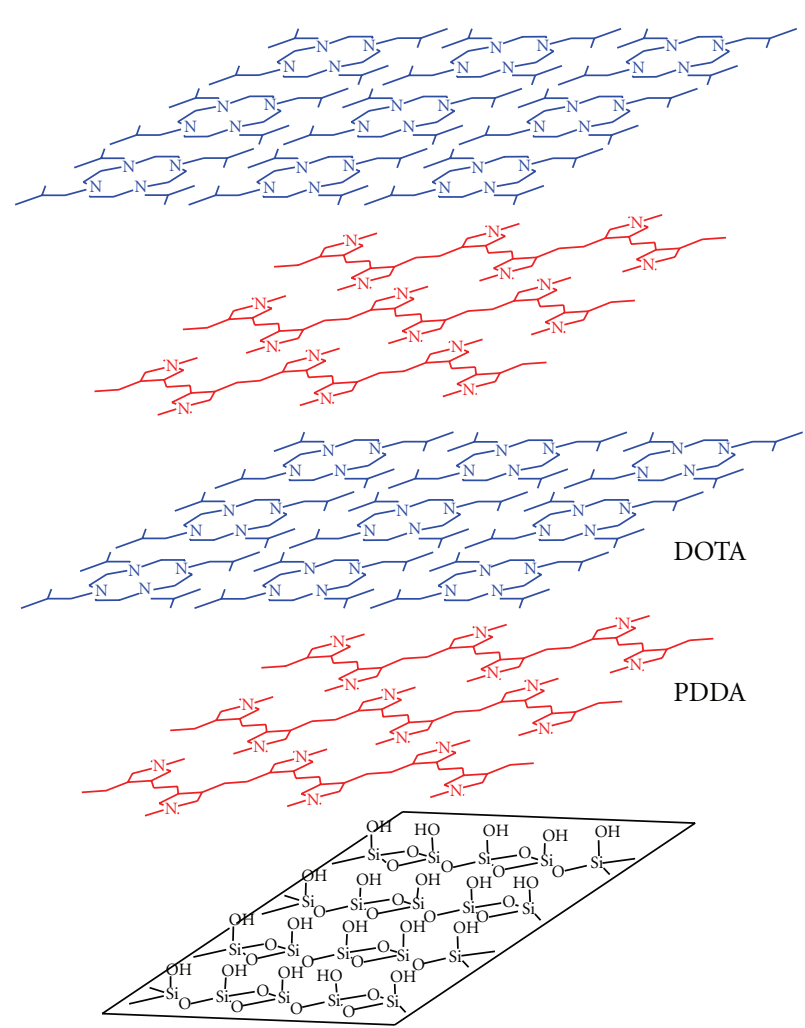

(b)

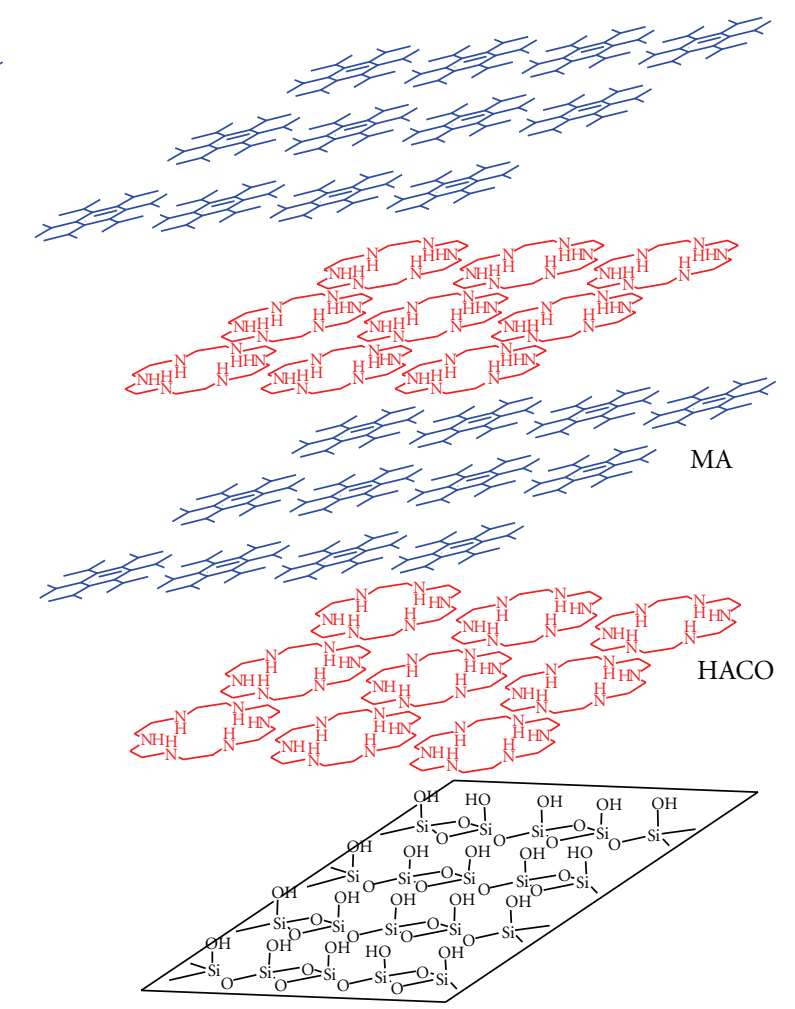

(d)

Figure 3: The visualization of PDDA and CuPc (a), PDDA and DOTA (b), PDDA and MA (c), and HACO and MA (d) ionic thin-film structures obtained from $\mathrm{X}$-ray results. 
ionic SAMs in an ordered way. A considerable delocalization of charge in the following layers is the condition of the ionic SAMs formation. These layers form a self-healing ordered ionic structure. The thicknesses of bilayers obtained from Xray measurements indicate that the molecules are laid in flat layers.

\section{References}

[1] G. Decher and J. D. Hong, "Buildup of ultrathin multilayer films by a self-assembly process: I. Consecutive adsorption of anionic and cationic bipolar amphiphiles on charged surfaces," Makromolekulare Chemie, Macromolecular Symposia, vol. 46, pp. 321-327, 1991.

[2] G. Decher and J. D. Hong, "Buildup of Ultrathin Multilayer Films by a Self-Assembly Process: II. Consecutive Adsorption of Anionic and Cationic Bipolar Amphiphiles and Polyelectrolytes on Charged Surfaces," Berichte der Bunsen-Gesellschaft für Physikalische Chemie, vol. 95, pp. 1430-1434, 1991.

[3] J. A. Jaber and J. B. Schlenoff, "Recent developments in the properties and applications of polyelectrolyte multilayers," Current Opinion in Colloid and Interface Science, vol. 11, no. 6, pp. 324-329, 2006.

[4] X. Zhang, H. Chen, and H. Zhang, "Layer-by-layer assembly: from conventional to unconventional methods," Chemical Communications, no. 14, pp. 1395-1405, 2007.

[5] M. Schönhoff, "Self-assembled polyelectrolyte multilayers," Current Opinion in Colloid and Interface Science, vol. 8, no. 1-2, pp. 86-95, 2003.

[6] P. T. Hammond, "Recent explorations in electrostatic multilayer thin film assembly," Current Opinion in Colloid and Interface Science, vol. 4, no. 6, pp. 430-442, 1999.

[7] F. Gröhn, "Electrostatic self-assembly as route to supramolecular structures," Macromolecular Chemistry and Physics, vol. 209, no. 22, pp. 2291-2301, 2008.

[8] F. M. Menger and L. Shi, "Electrostatic binding among equilibrating 2-D and 3-D self-assemblies," Journal of the American Chemical Society, vol. 131, no. 19, pp. 6672-6673, 2009.

[9] Y. Liu, A. Wang, and R. O. Claus, "Layer-by-layer electrostatic self-assembly of nanoscale $\mathrm{Fe}_{3} \mathrm{O}_{4}$ particles and polyimide precursor on silicon and silica surfaces," Applied Physics Letters, vol. 71, no. 16, pp. 2265-2267, 1997.

[10] A. Baba, Y. Kanetsuna, S. Sriwichai et al., "Nanostructured carbon nanotubes/copper phthalocyanine hybrid multilayers prepared using layer-by-layer self-assembly approach," Thin Solid Films, vol. 518, no. 8, pp. 2200-2205, 2010.

[11] S. Zhang, Y. Shao, G. Yin, and Y. Lin, "Electrostatic selfassembly of a pt-around-au nanocomposite with high activity towards formic acid oxidation," Angewandte ChemieInternational Edition, vol. 49, no. 12, pp. 2211-2214, 2010.

[12] S. Zhang, Y. Shao, G. Yin, and Y. Lin, "Carbon nanotubes decorated with Pt nanoparticles via electrostatic self-assembly: a highly active oxygen reduction electrocatalyst," Journal of Materials Chemistry, vol. 20, no. 14, pp. 2826-2830, 2010.

[13] V. Sivagnanam, A. Sayah, and M. A. M. Gijs, "Bead-based single protein micro-array realized through electrostatic selfassembly of carboxylated beads," Microelectronic Engineering, vol. 85, no. 5-6, pp. 1355-1358, 2008.

[14] T. Kobayashi, H. Fu, Q. Cui, and H. Wang, "Multilayer composite surfaces prepared by an electrostatic self-assembly technique with quaternary ammonium salt and poly(acrylic acid) on poly(acrylonitrile-co-acrylic acid) membranes," Journal of Applied Polymer Science, vol. 110, no. 5, pp. 3234-3241, 2008.

[15] F. Caruso, H. Lichtenfeld, M. Giersig, and H. Mohwald, "Electrostatic self-assembly of silica nanoparticle-polyelectrolyte multilayers on polystyrene latex particles," Journal of the American Chemical Society, vol. 120, no. 33, pp. 8523-8524, 1998.

[16] S. L. Craig, "From ionic liquids to supramolecular polymers," Angewandte Chemie - International Edition, vol. 48, no. 15, pp. 2645-2647, 2009.

[17] M. Lütt, M. R. Fitzsimmons, and D. Li, "X-ray reflectivity study of self-assembled thin films of macrocycles and macromolecules," Journal of Physical Chemistry B, vol. 102, no. 2, pp. 400-405, 1998.

[18] S.-H. Kim, C.-H. Ahn, S.-Y. Park, C.-J. Shin, and H.-J. Suh, "Electrostatic layer-by-layer self-assembly of anionic squarylium and cationic polyelectrolyte," Dyes and Pigments, vol. 69, pp. 108-110, 2006.

[19] X. Wang, J. Fang, D. Wang, and W. Zheng, "Multilayer films consisted of azulene-based dye molecules and polyelectrolyte: Preparation, characterization and photoluminescent property," Thin Solid Films, vol. 517, no. 24, pp. 6497-6501, 2009.

[20] S. K. Kumar, J. K. Park, and J. D. Hong, "Electrostatic selfassembly of a macrocyclic amphiphile for inducing the flat orientation of chromophores," Langmuir, vol. 23, no. 9, pp. 5093-5096, 2007.

[21] A. Tronin, Y. Lvov, and C. Nicolini, "Ellipsometry and xray reflectometry characterization of self-assembly process of polystyrenesulfonate and polyallylamine," Colloid \& Polymer Science, vol. 272, no. 10, pp. 1317-1321, 1994.

[22] J. Serafińczuk, J. Pietrucha, G. Schroeder, and T. P. Gotszalk, "Thin film thickness determination using X-ray reflectivity and Savitzky-Golay algorithm," Optica Applicata, vol. 41, no. 2, pp. 315-322, 2011.

[23] T. Gunnlaugsson and J. P. Leonard, "Responsive lanthanide luminescent cyclen complexes: from switching/sensing to supramolecular architectures," Chemical Communications, no. 25, pp. 3114-3131, 2005.

[24] R. W. Hay, M. T. H. Tarafder, and M. M. Hassan, "Copper(II) and nickel(II) complexes of hexacyclen $(1,4,7,10,13,16$-hexaazacyclo-octadecane) and the kinetics of dissociation of the copper(II) and nickel(II) complexes in acidic solution," Polyhedron, vol. 15, no. 4, pp. 725-732, 1996.

[25] J. Cullinane, R. I. Gelb, T. N. Margulis, and L. J. Zompa, "Hexacyclen complexes of inorganic anions: bonding forces, structure, and selectivity," Journal of the American Chemical Society, vol. 104, no. 11, pp. 3048-3053, 1982.

[26] A. C. Tedesco, J. C. G. Rotta, and C. N. Lunardi, "Synthesis, photophysical and photochemical aspects of phthalocyanines for photodynamic therapy," Current Organic Chemistry, vol. 7, no. 2, pp. 187-196, 2003.

[27] T. Inabe and H. Tajima, "Phthalocyanines_-versatile components of molecular conductors," Chemical Reviews, vol. 104, no. 11, pp. 5503-5533, 2004. 

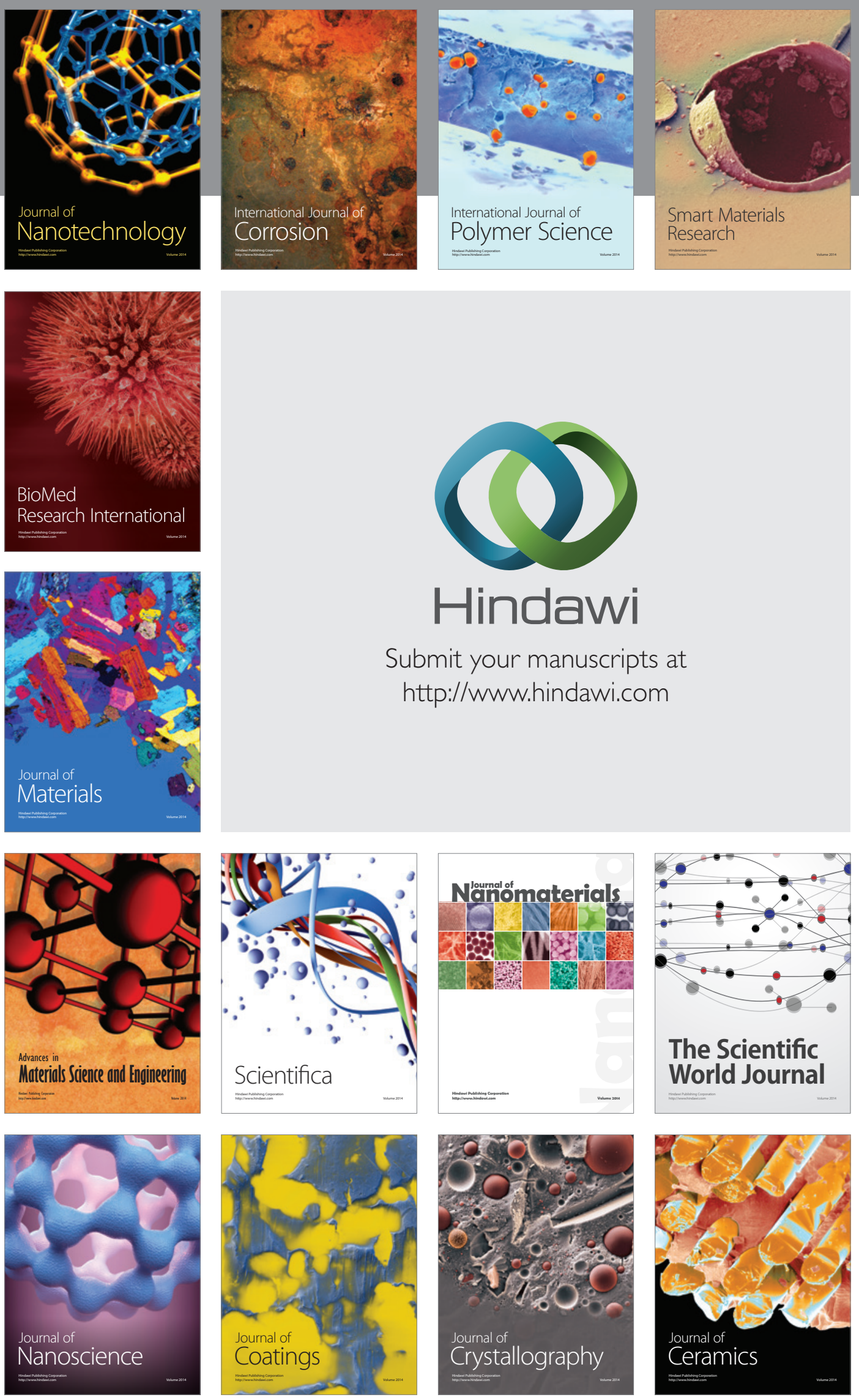

The Scientific World Journal

Submit your manuscripts at

http://www.hindawi.com

\section{World Journal}

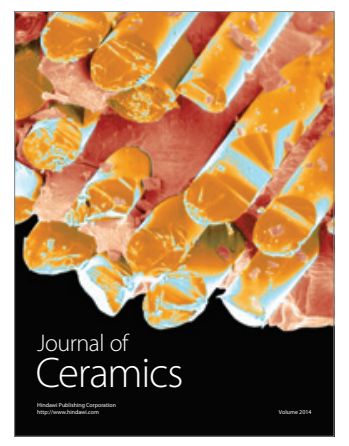

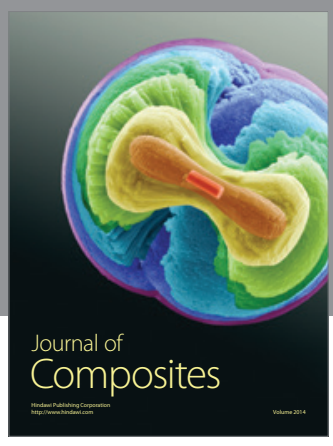
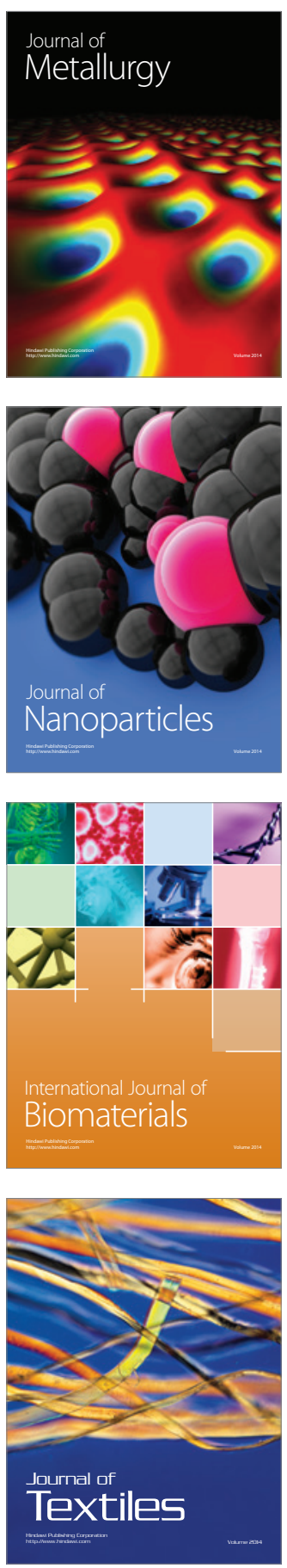